\title{
Nacktes Recht, abgeschriebene Tradition. Anmerkungen zu Reut Paz und den Herausforderungen des Rechtspluralismus
}

verfassungsblog.de/rechtspluralismus-und-seine-grundlagen-anmerkungen-zu-reut-paz/

Alexandra Kemmerer So 12 Aug 2012

So 12 Aug

2012

Unlängst hat Reut Yael Paz hier kritisch Stellung genommen

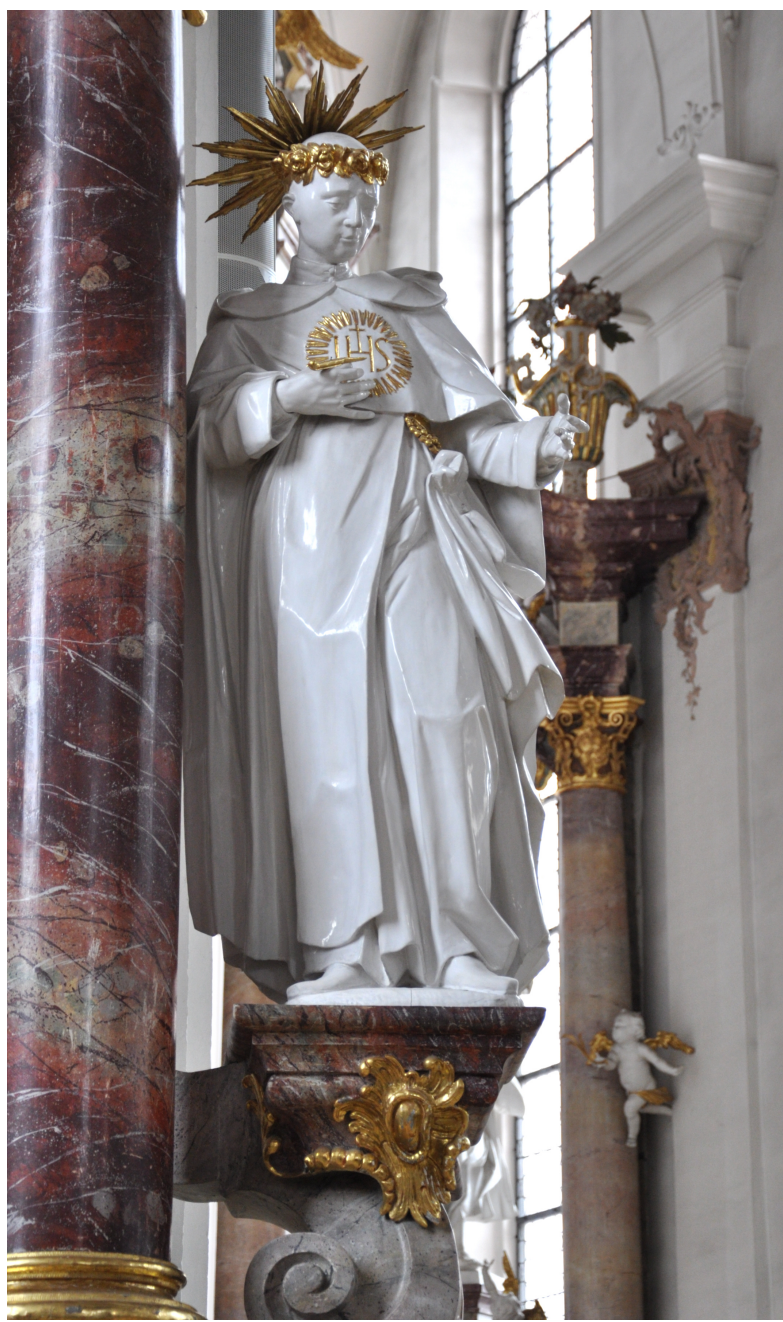

Zutreffend weist sie darauf hin, dass das Gericht die Herausforderungen des Rechtspluralismus und des im konkreten Fall vorliegenden Konfliktes divergierender normativer Ordnungen verkannt hat - und dass auch die bisherige Debatte diese Fragen nur unzureichend zur Sprache bringt.

Unklar und problematisch bleibt jedoch ihre eigene Unterscheidung zwischen Öffentlichem und Privatem. Gehört Religion nur in den Raum des Privaten? Abgesehen davon, dass das Gericht diese Differenzierung nicht explizit thematisiert, bleibt bei Reut Paz auch im Dunkeln, wie sich die Unterscheidung Öffentlich / Privat zu den konfligierenden normativen Ordnungen verhält. Und: Ist das Verhältnis zwischen der Verfassung und Ordnungen religiösen Rechts aus Sicht eines staatlichen Gerichts nicht notwendig ein asymmetrisches? (Was natürlich nicht heißt, dass nichtstaatliche Rechtsordnungen schlichtweg ignoriert werden können.)

Mehr als diese Fragen, die inzwischen von einigen Kommentatoren diskutiert wurden und auch andernorts besprochen werden, beschäftigt mich indessen ein von Reut Paz erhobener Vorwurf, der mir unhaltbar scheint und mich veranlasst, trotz anderer drängender Tagesaktualitäten noch einmal auf die Diskussion zurückzukommen. Reut Paz

eröffnet ihren Beitrag mit einer furiosen postkolonialen Ouvertüre, die den Ton vorgibt, in dem sie wenig später eine zentrale Frage des Beitrags formuliert: "Does this judgement reflect remnants of the European colonial/civilizing project in its full glory because it is linked to racial discrimination and a strong Christian (strike out the Judeo-) religious bias?"

Die Frage wird affirmativ beantwortet: "Although this situation is a classic example of the need to resolve conflicts between normative orders that need to coexist in the same social field (...) the court - without the necessary reflections in the court but also beyond - exclusively based its reasoning (on) the German legal system, a system which in this case also coincides with the Christian understanding of the physical body."

An dieser Stelle muss man Reut Paz, die eine hervorragende Erläuterung der Entscheidung des israelischen Supreme Court zur Brith Milah und ihres religionsrechtlichen Kontextes bietet, undifferenzierte Oberflächlichkeit bei der Analyse der Kölner Entscheidung vorhalten. Die Kölner Richter argumentieren völlig religionsblind und erweisen sich dabei als so geschichts- und gesellschaftsvergessen wie die strafrechtlichen Wortführer der Debatte. Sie machen sich nicht etwa ein "christliches Verständnis körperlicher Integrität" zu eigen, sondern bringen einen "religionsfeindlichen Zeitgeist" zum Ausdruck, den Patrick Bahners in der Beschneidungsdebatte 
gerade auch im Internet rabiat zum Durchbruch gekommen sieht.

Warum aber lässt sich der auf den ersten Blick religionsfeindlich-laizistische Zugriff der Kölner Richter nicht doch als untergründig christlich eingefärbte Voreingenommenheit klassifizieren, als gefügige Anpassung an eine christliche "Leitkultur"? Lässt sich da nicht etwas Klandestin-Christliches zwischen den Zeilen lesen? Leben wir nicht, zumal im Rheinland, in einer "immer noch" zutiefst christlich imprägnierten Gesellschaft?

Um diese Frage zu beantworten, braucht es ein kulturhistorisch-theologisches Wissen, das in unseren religionsverfassungsrechtlichen Debatten spätestens seit der Kruzifix-Entscheidung des

Bundesverfassungsgerichts nur noch als Leerstelle vorhanden ist. Die Repräsentanten der christlichen Kirchen übersetzen kantige theologische Argumente in allgemeines zivilgesellschaftliches Wortgeplänkel und üben sich (von Christoph Möllers gerade zurecht beklagt) mit mäßiger Überzeugungskraft als Grundrechtsinterpreten oder geben Themen mit gesellschaftspolitischem Konfliktpotential lieber gleich an Experten des Staatskirchenrechts ab.

Für die Katholikin ist das beschämend und bedauerlich - für die Juristin und Bürgerin der civitas terrena aber geradezu katastrophal. Denn der gelingende Umgang mit den Herausforderungen und Problemen des Rechtspluralismus lebt von Voraussetzungen, die das Recht und die Rechtswissenschaft selbst nicht bereitstellen und garantieren können. "Der Rechtspluralismus ist die große Herausforderung für die Einheit des öffentlichen Rechts, hier liegen die Zukunftsaufgaben der Wissenschaft, die sich nur mit Kontextualisierung Iösen lassen und damit auf die Grundlagenfächer verweisen“, konstatiert Dieter Grimm, und bringt damit das Dilemma auf den Punkt. Wo das kompetitive, aber auch komplementäre Neben- und Miteinander verschiedener Rechtssysteme und normativer Ordnungen Teil des sozialen Alltags geworden ist, braucht es Kontextwissen, das nur von außen in den Raum des Rechts vermittelt werden kann. Von den "Nachbarwissenschaften", deren Diskurse idealerweise über die Grundlagenfächer in die Rechtswissenschaft hinein "übersetzt" werden, aber auch von Vertretern religiöser und nichtreligiöser normativer Ordnungen. In den Gesellschaften der Gegenwart braucht es, wie Charles Taylor und Jocelyn Maclure es unlängst gefordert haben, die Entwicklung eines historisch fundierten "ethischen und politischen Wissens", das Toleranz und Solidarität ermöglicht.

In der Debatte um die Beschneidung ist das Angebot solchen Kontextwissens spärlich. Während man vielerorts bis ins Kleinste die medizinischen Details des Eingriffs nachlesen konnte, blieben theologische und historische Kommentare rar, nicht nur aus christlicher Feder. So hätte man sich von einem Experten für jüdische Geschichte und Kultur einige Anmerkungen zur Diskussion um die Geltung und Anwendung Jüdischen Rechts im Deutschland des 19. Jahrhunderts gewünscht. Reut Paz' Hinweise auf die israelische Rechtsprechung und deren Umgang mit religionsrechtlichen Argumenten füllen eine andere in der Diskussion zuvor kaum beachtete Lücke.

Doch warum liegt sie falsch mit ihrer Unterstellung einer "strong Christian (...) religious bias"?

Weil auch das christliche Verständnis körperlicher Integrität von Traditionen geprägt ist, die dem laizistischen Humanisten eine archaische Zumutung sein müssen. Keineswegs wird der Körper als von Geburt an vollkommen und damit in seiner Integrität als unantastbar verstanden. Die Konditionierung des Körpers durch Askese, die imitatio Christi durch Verzicht und Disziplinierung des Körpers ist religionsgeschichtlich ein zentraler Aspekt christlichen Glaubens (wie der meisten anderen Religionen). In der Geschichte des Christentums reicht die Bandbreite der Praktiken einer "Vervollkommnung" des Körpers vom moderaten Fasten (protestantischneudeutsch: "sieben Wochen ohne") bis hin zu blutigen Eingriffen in den eigenen Körper, die uns befremdlich anmuten - man denke an die Stigmata des heiligen Franziskus von Assisi oder an den Mystiker Heinrich Seuse, der sich im 14. Jahrhundert das Monogramm des Namens Jesu in die Haut über dem Herzen ritzte, als Zeichen der ewigen Verbundenheit zwischen Gott und Mensch (als "Vorläufer des modernen Tattoo" haben Wissenschaftler der Uni Heidelberg diese inbrünstig-blutrünstige Minnepraxis jüngst charakterisiert).

Das Minnezeichen auf Seuses Haut ist, wie die reichen kulturwissenschaftlich-theologischen Erläuterungen der "Poetischen Dogmatik" des Kölner Theologen Alex Stock nahelegen, als Namenszeichen in subtiler Weise mit dem von Reut Paz erwähnten Fest der Beschneidung Jesu verbunden, das die Kirche in der außerordentlichen Form ihres Ritus zugleich als Fest der Namensgebung Jesu feiert. Man denkt an die von Mose und Jeremia wie 
von Paulus beschworene "Beschneidung des Herzens". Offenkundig endet die Geschichte des Christentums mit der Beschneidung nicht bei Paulus (auch wenn man das dem gut protestantisch ausschließlich auf die Bibel rekurrierenden bayerischen "Sonntagsblatt" so entnehmen könnte), und spätere Traditionslinien lassen sich auch nicht nur auf antisemitische Ressentiments beschränken. Im Gegenteil: es gibt für Christen gute theologische Gründe, sich für den Schutz einer Beschneidungspraxis stark zu machen, die ihnen selbst nicht geboten ist.

Hier weiter auszuholen wäre aber die Sache von Theologen, Historikern und Kulturwissenschaftlern. Oder, noch besser, von historisch und kulturwissenschaftlich beschlagenen Theologen. Dass die forcierte Beschäftigung mit der eigenen Tradition jedenfalls im Katholizismus derzeit oft mit Modernismuskritik und intellektuellenfeindlichem Ressentiment einhergeht, stimmt die im Angesicht des Rechtspluralismus kontextinteressierte Juristin leider nicht besonders optimistisch, wenn sie auf die Erschließung neuer Wissensquellen hofft, die die christliche Prägung unserer Gesellschaft und ihrer normativen Ordnungen erhellen.

Dennoch: die Herausforderungen des Rechtspluralismus bekommen wir nur auf der Grundlage fundierten Wissens um religiöse und kulturelle Traditionen in den Griff. Wo die einfach als vorgestrig abgeschrieben werden und man geschichtsvergessen nur auf das nackte Recht setzt, ist der clash of cultures vorprogrammiert. Mit leichtfertigen Unterstellungen religiös-kultureller Voreingenommenheit droht uns tatsächlich, was manche Kommentatoren bereits beschwören: ein veritabler Kulturkampf.

Unser Bild zeigt die Figur des Mystikers Heinrich Seuse in der Predigerkirche Rottweil.

Foto: Andreas Praefcke, Creative Commons Licence (CC BY 3.0)

\section{LICENSED UNDER CC BY NC ND}

SUGGESTED CITATION Kemmerer, Alexandra: Nacktes Recht, abgeschriebene Tradition. Anmerkungen zu Reut Paz und den Herausforderungen des Rechtspluralismus, VerfBlog, 2012/8/12, http://verfassungsblog.de/rechtspluralismus-und-seine-grundlagen-anmerkungen-zu-reut-paz/. 